\title{
Transcranial Direct Current Stimulation Based Metaplasticity Protocols in Working Memory
}

\author{
Sandra Carvalho a,b,c,*, Paulo S. Boggio ${ }^{\text {, }}$, Óscar F. Gonçalves ${ }^{\text {a,b,c,e }}$, Ana Rita Vigário a , Marisa Faria ${ }^{\text {, }}$ \\ Soraia Silva ${ }^{\mathrm{a}}$, Gabriel Gaudencio do Rego ${ }^{\mathrm{d}}$, Felipe Fregni ${ }^{\mathrm{b}, \mathrm{c}}$, Jorge Leite ${ }^{\mathrm{a}, \mathrm{b}, \mathrm{c}}$ \\ ${ }^{a}$ Neuropsychophysiology Laboratory, CIPsi, School of Psychology (EPsi), University of Minho, Campus de Gualtar, 4710-057 Braga, Portugal \\ ${ }^{\mathrm{b}}$ Spaulding Neuromodulation Center, Department of Physical Medicine and Rehabilitation, Spaulding Rehabilitation Hospital, Harvard Medical School, Boston, MA, USA \\ ${ }^{\mathrm{c}}$ Department of Physical Medicine and Rehabilitation, Massachusetts General Hospital, Harvard Medical School, Boston, MA, USA \\ d Social and Cognitive Neuroscience Laboratory and Developmental Disorders Program, Center for Health and Biological Sciences, Mackenzie Presbyterian University, \\ 01241-001 Sao Paulo, Brazil \\ ${ }^{\mathrm{e}}$ Department of Counseling and Applied Educational Psychology, Bouvé College of Health Sciences, Northeastern University, Boston, USA
}

\section{A R T I C L E I N F O}

\section{Article history:}

Received 7 August 2014

Received in revised form

17 November 2014

Accepted 19 November 2014

Available online 27 December 2014

\section{Keywords:}

Working memory

N-Back

Metaplasticity

Pre-conditioning and conditioning tDCS

DLPFC

\begin{abstract}
A B S T R A C T
Background: It has been already shown that delivering tDCS that are spaced by an interval alters its impact on motor plasticity. These effects can be explained, based on metaplasticity in which a previous modification of activity in a neuronal network can change the effects of subsequent interventions in the same network. But to date there is limited data assessing metaplasticity effects in cognitive functioning. Objectives: The aim of this study was to test several tDCS-based metaplasticity protocols in working memory (WM), by studying the impact of various interstimulation intervals in the performance of a 3-back task.

Methods: Fifteen healthy volunteers per experiment participated in this study. Experiments 1 and 2 tested an anodal tDCS-induced metaplasticity protocol $\left(1 \mathrm{~mA}, 10+10^{\prime}\right)$ with 3 interstimulation intervals $(10,30$, and $60 \mathrm{~min})$. Experiment 3 determined the effects of a similar protocol-with a 10 -min interval between two sessions of cathodal tDCS or anodal plus cathodal tDCS $\left(1 \mathrm{~mA}, 10+10^{\prime}\right)$.

Results: Two consecutive sessions of anodal tDCS delivered with a 10 min interval between them did not improve WM performance $(P=.095)$. This effect remained the same if the interval was increased to 30 or $60 \mathrm{~min}$. In contrast, when a $10 \mathrm{~min}$ interval was given between two consecutive cathodal tDCS sessions, performance in the 3 back task increased $(P=.042)$.

Conclusions: These results suggest that the polarity effects of tDCS on working memory are dependent on the previous level of activity of the recruited neural population.
\end{abstract}

(c) 2015 Elsevier Inc. All rights reserved.

\section{Introduction}

Electrical stimulation has been used as a tool to modulate human plasticity. Our understanding of how electrical stimulation

Conflict of interest: The authors do not have any conflicts of interest, financial or otherwise, to disclose.

This work was supported by the Portuguese Foundation for Science and Technology (FCT) with two individual grants (SFRH/BPD/86041/2012 and SFRH/BPD/ $86027 / 2012)$.

* Corresponding author. Neuropsychophysiology Laboratory, CIPsi, School of Psychology (EPsi), University of Minho, Campus de Gualtar, 4710-057 Braga, Portugal.

E-mail address: sandrarc@psi.uminho.pt (S. Carvalho). shapes the organization of the human brain has guided the development of cognitive enhancement protocols. One cognitive domain that is modulated by electrical stimulation is working memory (WM). WM is defined as the ability to maintain and manipulate information online for short periods [1,2]. Several studies have investigated the effects of various transcranial direct current stimulation (tDCS) protocols on working memory [3-7].

In tDCS, a weak constant electric current is used with at least 2 electrodes: anodal (positive pole) and cathodal (negative pole). Anodal tDCS is associated with a depolarizing effect on the neural membrane, whereas cathodal tDCS hyperpolarizes it $[8,9]$. This initial effect on the properties of the neuronal membrane leads to secondary changes in plasticity by increasing decreasing spontaneous neuronal activity [10]. It is possible to enhance WM using 
anodal tDCS over the dorsolateral prefrontal cortex (DLPFC) $[3,6,7,11]$. These effects are time-dependent and can persist for at least 30 min after tDCS has ended [5].

Recently, the effects of tDCS on cortical plasticity have been shown to depend on the duration and interstimulation interval. Monte-Silva et al. noted that delivering tDCS in consecutive sessions that are spaced by an interval alters its impact on motor plasticity [12]. These effects can be explained, based on metaplasticity in which a previous modification of the activity in a neuronal network can impact the effects of subsequent interventions to the same network [13]. Thus, tDCS allows us to assess the effects of metaplasticity if a second session of tDCS is delivered during the effects of the previous one. To this end, we were interested in determining these effects using working memory as a surrogate for cognitive plastic changes.

In order for metaplasticity to occur, tDCS stimulation needs to be paused and the second tDCS session (i.e., conditioning tDCS) needs to be delivered during the after effects of the first one (i.e., pre-conditioning tDCS).

Our aim was to examine the effects of metaplasticity on working memory by studying the effects of consecutive sessions of tDCS with various interstimulation intervals. Experiments 1 and 2 tested continuous anodal tDCS and discontinuous anodal tDCS using several interstimulation intervals (i.e. 10, 30 and $60 \mathrm{~min}$ ). Experiment 3 tested a similar protocol with an interstimulation interval, instead using cathodal tDCS as pre-conditioning and 2 polarities as conditioning stimulation: anodal or cathodal tDCS. We hypothesized that introducing an interval between 2 short sessions of anodal tDCS would enhance its impact on working memory-an effect that could be characterized by potentiation or temporal summation, similar to what is observed with cathodal tDCS in the motor cortex [12].

\section{Methods}

\section{Participants}

Forty-five healthy volunteers (15 per experiment) were enrolled in this study. In experiment 1, 15 undergraduate students from University of Minho volunteered (12 females; $20.2 \pm 2.7$ years old). Experiment 2 comprised 15 undergraduate students from Mackenzie University ( 8 females; $21.5 \pm 2.6$ years old). In experiment 3 , 15 undergraduate students from University of Minho volunteered (14 females; $20.1 \pm 1.8$ years old).

All participants were right-handed and healthy, with normal or corrected-to-normal visual acuity and no current or past history of neurological or psychiatric disorders. Participants were excluded if any medication or psychotropic drugs had been used in the 4 weeks prior to the study. Participants were advised to avoid alcohol, cigarettes, and caffeinated drinks on the day of the experiment, and none reported fatigue due to insufficient sleep.

All participants gave written informed consent prior to study inclusion. The study was approved by the local ethics committee and was conducted per the Declaration of Helsinki.

\section{Design}

Each experiment consisted of 3 sessions, with an intersession interval of at least 1 week. The experimental design of each session comprised 3 blocks: 1) pre-conditioning tDCS; 2) Interval; and 3) Conditioning tDCS, with the experimental task on the last $5 \mathrm{~min}$. The 3 experiments are described below (Fig. 1):

- Experiment 1 (10-min interval): The goal of this experiment was to determine the effects of a 10 -min interval $\left(10^{\prime} \mathrm{i}\right)$ between the first and second consecutive anodal tDCS sessions compared with 2 control conditions. The 3 conditions were: 1) anodal tDCS-10'i-anodal tDCS (10-min interval with anodal tDCS), 2) rest - anodal tDCS-anodal tDCS (control condition 1, no interval with anodal tDCS), and 3) rest - sham tDCS-sham tDCS (control condition 2, sham tDCS only).

- Experiment 2 (30- and 60-min intervals): The goal of this experiment was to test longer intervals between consecutive anodal tDCS sessions. The design was the same as in experiment 1 , except with $30^{\prime}$ and $60^{\prime}$ intervals and the respective sham conditions. Namely, the conditions were: 1 ) anodal tDCS - 30'i - anodal tDCS (30-min interval with anodal tDCS), 2) sham tDCS - 30' $\mathrm{i}$ - sham tDCS (control condition 1, sham tDCS only with a $30^{\prime}$ interval), 3) anodal tDCS - 60'i-anodal tDCS (60-min interval with anodal tDCS), 2) sham tDCS - 60'i - sham tDCS (control condition 2, sham tDCS only with a $60^{\prime}$ interval). Two sham conditions were included in order to increase blinding, due to the different interstimulation interval.

- Experiment 3 (10-min interval with cathodal stimulation): In this experiment, we examined whether cathodal tDCS in the pre-conditioning block alters the effects of metaplasticity, testing 3 conditions: 1 ) cathodal tDCS-10'i-anodal tDCS (10-min interval with cathodal and anodal), 2) cathodal tDCS-10' $\mathrm{i}-$ cathodal tDCS (10-min interval with cathodal and cathodal), and 3) sham tDCS-10'i-sham tDCS (control condition with sham tDCS) (Fig. 1).

Task

The 3-back task was adapted from Fregni et al. [3], in which participants were instructed to respond "Y" (yes) if a letter that appeared on the center of a screen (i.e., target) was the same as the one that flashed 2 letters earlier or " $N$ " (no) if it was not. There were 30 "Y" and 165 "N" responses, totaling 195 trials. Each letter appeared for $30 \mathrm{~ms}$, separated by a 2000-ms intertrial interval (ITI). The order of the letters was randomized, thus reshuffling the actual targets between sessions and preventing memorization effects to be carried over from one tDCS session to the next. This was done in a manner that for each experiment, the 195 trials sequence was randomly generated. Therefore, the 30 "Y" targets were generated for that specific sequence, based on the 3 trials earlier match rule.

\section{Transcranial direct current stimulation (tDCS)}

tDCS ( $1 \mathrm{~mA}$ ) was applied using $35-\mathrm{cm}^{2}$ saline-soaked electrode sponges. For experiments 1 and 3, an Eldith DC Stimulator Plus (Neuroconn, Germany) was used, whereas a locally developed DC stimulator was used for experiment 2 (contact psboggio@gmail. com for technical details).

Each experiment had a within-subject design, in which all participants were subjected to 3 (4 in experiment 2) tDCS conditions. The active electrode (anode or cathode) was placed over the left DLPFC, and the return electrode (cathode or anode) covered the contralateral supraorbital area (F3 and Fp2 electrode sites, respectively) [14]. Anodal or cathodal tDCS (1 mA) were applied in blocks of 10 min (with a 15-s ramp up and down), with the exception of the no interval anodal tDCS condition (experiment 1), which was applied for 20 min consecutively (with 15-s ramp up and down). Sham tDCS was applied with $1 \mathrm{~mA}$ intensity during $15 \mathrm{~s}$ (with 15-s ramp up and down). Therefore the total duration of active tDCS ( $1 \mathrm{~mA}$ ) was 20 min (i.e. pre-conditioning plus conditioning) and $30 \mathrm{~s}$ for sham tDCS (i.e. pre-conditioning plus conditioning). The conditioning tDCS in the task block began 5 min before the actual task and continued for the entire duration of the task (5 min). 

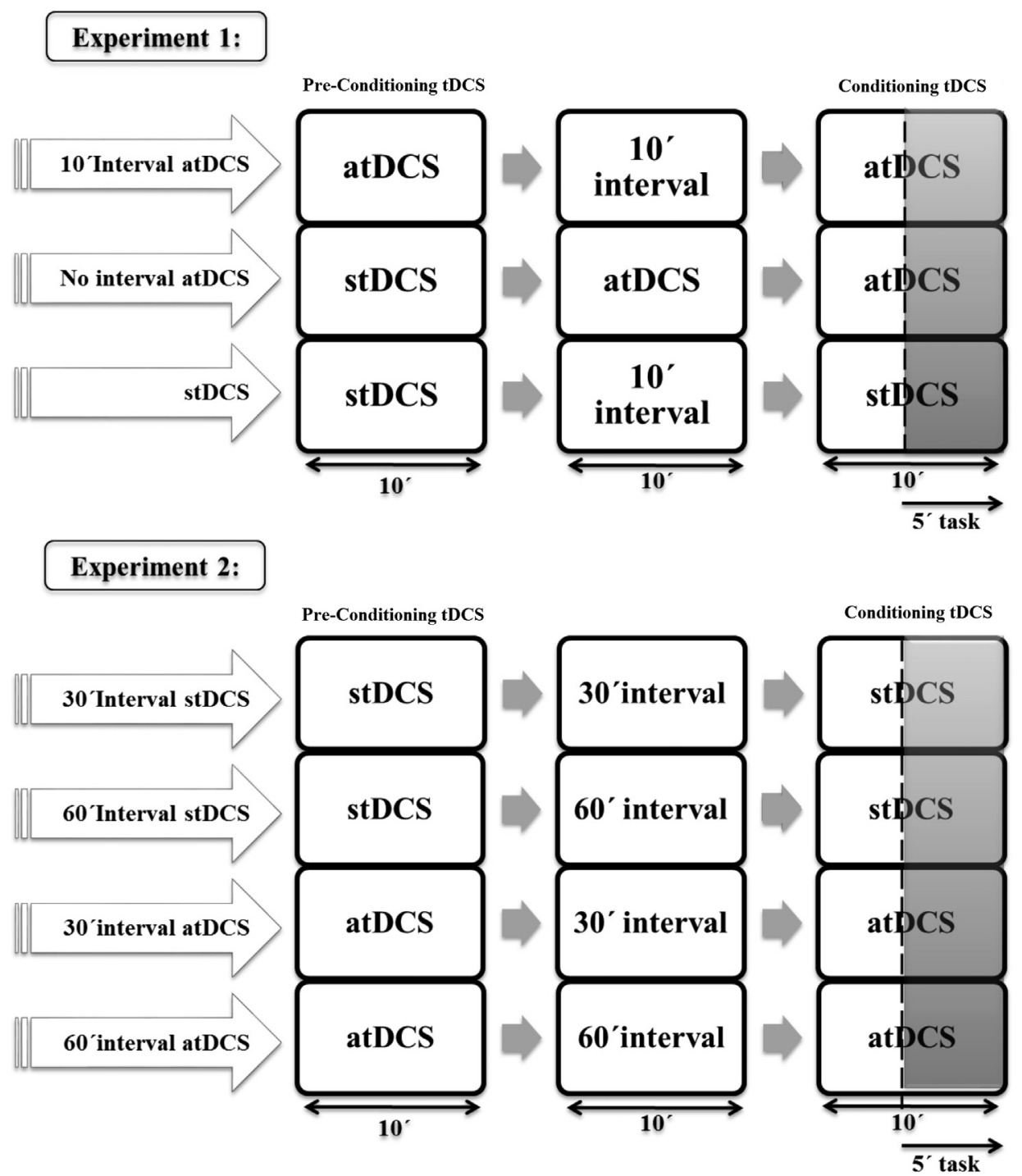

\section{Experiment 3:}
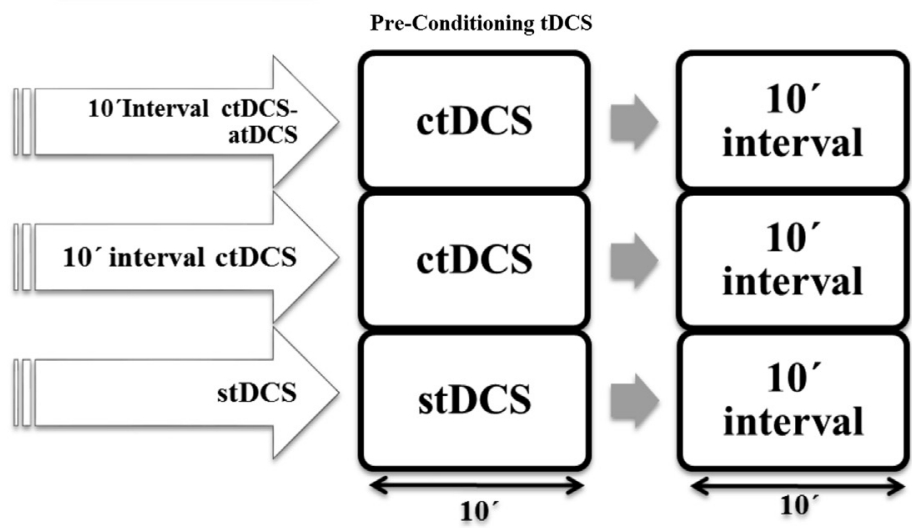

Conditioning tDCS

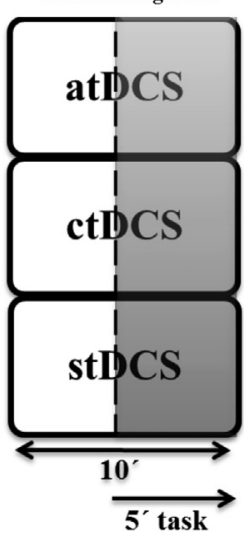

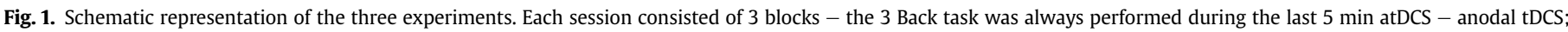
ctDCS - cathodal tDCS; stDCS - sham tDCS.

To prevent carryover effects, the sessions were separated by 1 week. The order in which tDCS condition was applied to each participant was randomized and counterbalanced in each experiment.

\section{Data analysis}

The effects of conditioning tDCS on working memory in the 3-back task were measured as the percentage of correct (i.e., "Y") 
responses. Each experiment was analyzed using a repeated measures general linear model ANOVA with tDCS protocol as the factor (3 levels for experiments 1 and 3; and 4 levels for experiment 2).

One-way independent sample ANOVA was performed to compare the performance of participants between experiments in the sham condition (with 3 levels, one for each experiment). Three separate one-way repeated-measures ANOVAs were performed to analyze the effects of tDCS over response bias. In the experiments where tDCS increased significantly WM performance, an additional repeated measures ANOVA with session order as factor was performed, in order to control for possible learning effects. When sphericity was not met, Greenhouse-Geisser correction was applied to the degrees of freedom in all cases with the corrected probabilities. Post hoc comparisons of the mean values were conducted by paired multiple comparison (with Bonferroni correction for multiple comparison) when the ANOVAs indicated significant effects. The criterion for statistical significance was $P<.05$. All statistical analyses were performed with SPSS for Windows (version 21.0.0, IBM, US).

\section{Results}

No adverse effects were reported in any experiment.

\section{Experiment 1 (10-min interval-anodal tDCS)}

One participant was removed from the analysis, because he did not complete all 3 tDCS conditions.

There was a significant main effect of tDCS protocol $[F(2,28)=8.760, P=.001]$. As expected, no interval anodal tDCS (active control) significantly increased the number of correct responses $(M=74.666, \mathrm{SE}=3.590)$ compared with sham $(M=64.000$, $\mathrm{SE}=3.838)(P \leq 001)$. The 10 -min interval tDCS condition did not significantly affect performance $(M=69.777, \mathrm{SE}=3.372)$ versus control sham $(P=.095)$ (Fig. 2). There were no significant effects of session order on working memory performance $[F(2,28)=.116$, $P=$.891].

\section{Experiment 2 (30- and 60-min interval experiment)}

All 15 participants performed all conditions. One participant was removed from the analysis due to an accuracy score of less than $25 \%$.

The 30- and 60-min intervals did not elicit any significant differences compared with sham tDCS - there was no significant main effect of tDCS protocol $[F(3,39)=.351, P=.789]$ (Fig. 2 ).

\section{Experiment 3 (10-min interval with cathodal stimulation)}

Two participants were removed from the analysis due to accuracy scores of less than $25 \%$.

There was a significant main effect of tDCS protocol $[F(2,24)=5.818, P=.009]$. In the post hoc Bonferroni-corrected pairwise comparisons, the 10 -min interval with cathodal tDCS $(M=71.026, \mathrm{SE}=4.019)$ significantly increased the percentage of hits versus sham $(M=63.590, \mathrm{SE}=4.928)(P=.042)$ and the $10-\mathrm{min}$ interval condition with opposite polarity (cathodal and anodal) $(M=61.026, \mathrm{SE}=4.001)(P=.012)$ (Fig. 2). There were no significant effects of session order on working memory performance $[F(2,24)=.022, P=.878]$.

\section{Sham group analysis between groups}

The subjects perform identically between experiments-there were no significant differences in the percentage of hits across sham sessions $[F(2,41)=.271, P=.764]$. By paired sample $t$-test for experiment 2, the increase in the interval (from 30 to $60 \mathrm{~min}$ ) did not have any effects under the sham tDCS conditions $[t(13)=.193$, $P=$.850].

\section{Bias analysis}

To better our understanding of these effects, an additional measure of $\beta$ (decision bias) was assessed. There was no evidence of the effects of tDCS protocol with regard to decision bias in experiments $1[F(2,28)=.585, P=.564], 2[F(3,39)=.886, P=.397$, $\varepsilon=.484]$, or $3[F(2,24)=1,212, P=.315]$ (Fig. 3 ).

\section{Discussion}

The objective of this study was to test several tDCS-based metaplasticity protocols in working memory as assessed by performance in a 3-back task. In experiments 1 and 2, we examined 10-, 30-, and 60-min intervals between the pre-conditioning and conditioning anodal tDCS compared with sham stimulation. In experiment 3, we tested the effects of a $10^{\prime}$ interval protocol between consecutive sessions of tDCS, with cathodal tDCS as pre-conditioning and either anodal or cathodal tDCS as conditioning.

Overall, there were several main findings. (i) Using a metaplasticity protocol with anodal tDCS, no significant effects of subsequent anodal tDCS sessions on working memory performance were observed when compared to sham stimulation, regardless of the interval (i.e., 10,30 or $60 \mathrm{~min}$ ); (ii) the administration of

\section{Correct Responses}
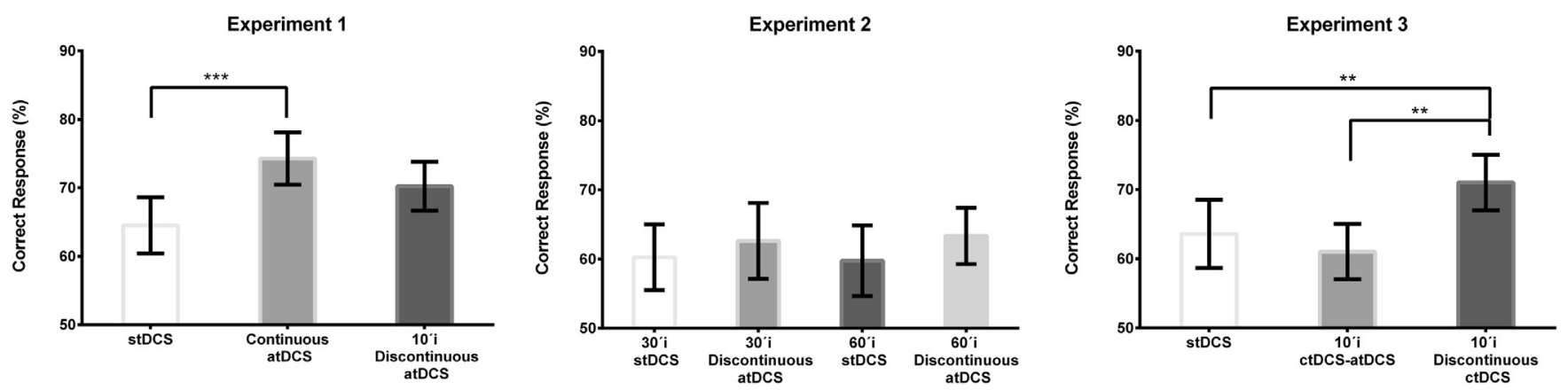

Fig. 2. Percentage of correct responses. The columns represent the mean percentage of correct responses (i.e. "Y") and the bars one SEM. ${ }^{* *} P<.01$; ${ }^{* * *} P<.001$. 


\section{Response preference $\beta$}

Experiment 1

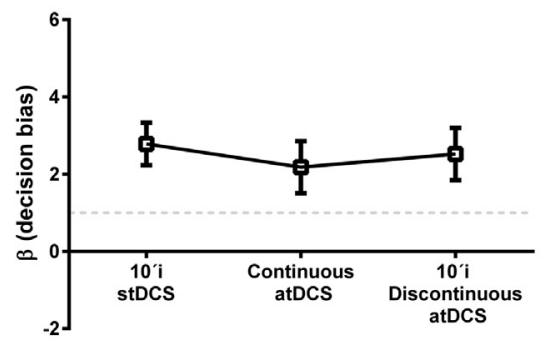

Experiment 2

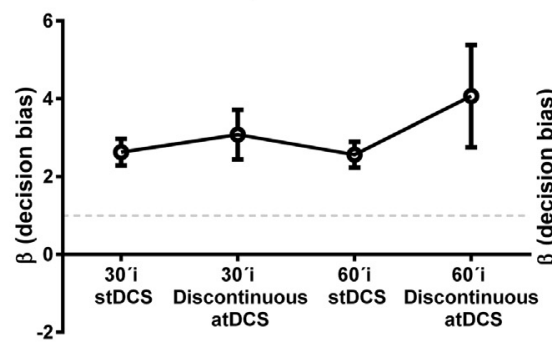

Experiment 3

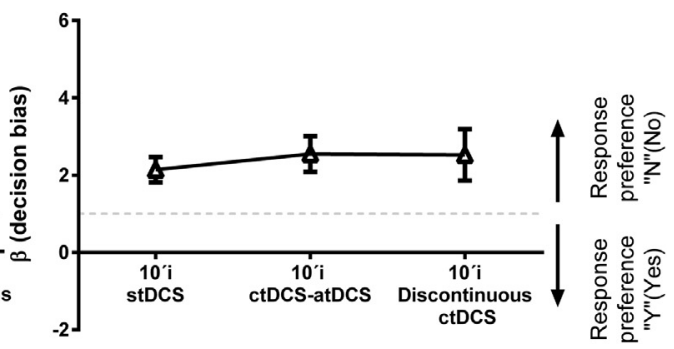

Fig. 3. Response preference. The bars represent one standard mean. Scores below 1 represent a response preference to Yes; scores above 1 represent a response preference to Yes.

continuous anodal tDCS (without this metaplasticity protocol) had a significant effect on working memory compared with sham stimulation; and (iii) the cathodal tDCS metaplasticity protocol significantly modulated the subsequent effects of cathodal tDCS on working memory, thus increasing working memory performance.

The findings of this study can be explained by the theory of metaplasticity. Our results support the bidirectional synaptic plasticity theory [15], which posits that the recent history of synaptic activity will impact ongoing activity. In other words, if synaptic activity has been already modulated by the preconditioning tDCS, delivering conditioning tDCS after a break can change the expected polarity effects thus interfering with the performance. Whereas continuous conditioning anodal tDCS positively impacted working memory, pre-conditioning stimulation with anodal tDCS mitigate the effects of subsequent anodal tDCS conditioning stimulation. Pre-conditioning of the underlying cortical region with anodal tDCS could have enhanced cortical activity through synaptic plasticity, which in turn might have interfered with the effects of conditioning anodal tDCS during task performance.

This is not the first time that such attenuation effects are observed. For instance, Huang et al. [16] reported that when rat hippocampus is primed with a short stimulus that induces shortterm potentiation and then conditioned with stronger stimulation [that can induce long-term potentiation (LTP)], LTP is no longer observed. This result is similar to our findings. It appears that the synaptic activity that was induced by pre-conditioning anodal tDCS interacted with conditioning anodal tDCS, generating a metaplasticity effect that down regulates task performance. Notably, this down regulating effect between anodal tDCS session was still evident even with a 60 interval between sessions (as can be seen in experiment 2). Although the duration of the after effects of anodal tDCS in the DLPFC has not been determined, studies on the human motor cortex have suggested that $10 \mathrm{~min}$ of anodal tDCS increase cortical excitability (i.e., induces aftereffects) for approximately $60 \mathrm{~min}[8,17]$. Our behavioral data showing lack of anodal tDCS effects on working memory after 60 min of preconditioned TDCS seems to suggest similar lengths for the aftereffects in the DLPFC, because as Fricke et al. [18] pointed out, in order to induce metaplasticity, the conditioning stimulation must be administered during the aftereffects of the pre-conditioning stimulation.

In our study, continuous anodal tDCS facilitated performance on the task compared with sham tDCS, which replicated the findings from other studies [3]. However, when an interval of 10,30 , or 60 min was introduced between the 2 consecutive anodal tDCS sessions a metaplasticity effect was observed. In this case, no changes in task performance were evident when comparing to sham tDCS. These results suggest attenuation [19] of the effect of anodal tDCS in WM performance. However, a significant positive effect in working memory performance was observed when conditioning cathodal tDCS, was primed by cathodal tDCS. Although we did not test the effect of continuous cathodal tDCS in working memory performance, previous studies failed to demonstrate such effects [3].

Two consecutive sessions of cathodal tDCS, with a $10^{\prime}$ interval between them, enhanced working memory performance, thus suggesting that the manipulation of the baseline physiologic state interferes with online neuromodulation. It has already been shown that pre-conditioning the neural network can induce homeostatic changes at the synaptic level [20]. It is possible that a compensatory up-regulation process occurs in the post-synaptic membrane receptors, as a response to previous inhibitory modulation, thus assuring that the neural functions are kept within optimal range $[13,15]$. If cortical excitability can be stabilized within a range by homeostatic plasticity mechanisms [21] it is possible that an initial down-regulation induced by cathodal tDCS was reverted by the conditioning cathodal tDCS. Thus rendering more excitable the task-related neural population, in what has been called the "rebound effect" [22].

Several other studies have been supporting this "rebound effect." For instance, high dosages of valproate, combined with $1 \mathrm{~Hz}$ rTMS, increase cortical excitability [23] and similar effects have been observed when $1 \mathrm{~Hz}$ rTMS is primed by cathodal tDCS stimulation [21]. These effects are believed to reflect homeostatic plasticity, wherein a physiologic state with decreased activity reacts to more inhibitory stimulation by reversing its state and thus increasing activity.

Pre-conditioning the conditioning anodal tDCS with cathodal tDCS did not significantly alter task-related performance, for which we expected metaplasticity effects. Previous studies showed that pre-conditioning the conditioning anodal tDCS with cathodal tDCS increases cortical excitability [19,24]. Nevertheless, we must distinguish cortical excitability from task performance. In the motor cortex, consecutive sessions of the same tDCS polarity initially decreased cortical excitability and then followed the same direction of the polarity of a single session but with a prolonged aftereffect $[12,25]$. However, in these studies cortical excitability was probed but without a clear relationship with behavioral performance. Simis et al. [26] found that $20 \mathrm{~min}$ of anodal tDCS enhanced motor performance following decreases in cortical excitability. Thus, there appears to be a nonlinear relationship between cortical excitability and behavioral performance. So in the present study it is possible that lack of behavioral effects was accompanied by changes at the cortical excitability level. Therefore, future studies should examine the link between cortical excitability and behavior, thus optimizing stimulation protocols. 
One potential limitation to the present results is that a different tDCS device was used for the second experiment. However experiments 1 and 2 are complementary as experiment 2 confirmed at some extent what was found in experiment 1 (i.e., adding an interval between anodal tDCS sessions has a negative behavioral impact on tDCS-induced effects).

Further, the ideal timing between tDCS sessions must be determined to establish the relationship between changes in excitability and behavioral performance. Also this timing can be critical, as it has been already demonstrated that homeostatic plasticity in the human motor cortex is time-dependent [18]. Our results suggest that inserting a short interstimulation interval between anodal tDCS sessions does not improve significantly working memory performance. Nonetheless, if a $10^{\prime}$ interstimulation interval is inserted between two cathodal tDCS sessions, then there is a significant increase in working memory performance, which suggests metaplasticity effects. Future studies should extend these findings and determine the effects on cortical excitability, testing various polarity combinations and with several interstimulation intervals.

\section{References}

[1] Baddeley A. Working memory. Science 1992;255(5044):556-9.

[2] Goldman-Rakic PS. Regional and cellular fractionation of working memory. Proc Natl Acad Sci U S A 1996;93(24):13473-80.

[3] Fregni F, Boggio PS, Nitsche M, et al. Anodal transcranial direct current stimulation of prefrontal cortex enhances working memory. Exp Brain Res 2005;166(1):23-30.

[4] Andrews SC, Hoy KE, Enticott PG, Daskalakis ZJ, Fitzgerald PB. Improving working memory: the effect of combining cognitive activity and anodal transcranial direct current stimulation to the left dorsolateral prefrontal cortex. Brain Stimul 2011;4(2):84-9.

[5] Ohn SH, Park C-I, Yoo W-K, et al. Time-dependent effect of transcranial direct current stimulation on the enhancement of working memory. Neuroreport 2008;19(1):43-7.

[6] Boggio PS, Ferrucci R, Rigonatti SP, et al. Effects of transcranial direct current stimulation on working memory in patients with Parkinson's disease. J Neurol Sci 2006;249(1):31-8.

[7] Jo JM, Kim Y-H, Ko M-H, Ohn SH, Joen B, Lee KH. Enhancing the working memory of stroke patients using tDCS. Am J Phys Med Rehabil 2009;88(5):404-9.

[8] Nitsche MA, Paulus W. Sustained excitability elevations induced by transcranial DC motor cortex stimulation in humans. Neurology 2001;57(10):1899-901.

[9] Nitsche MA, Nitsche MS, Klein CC, Tergau F, Rothwell JC, Paulus W. Level of action of cathodal DC polarisation induced inhibition of the human motor cortex. Clin Neurophysiol 2003;114(4):600-4.
[10] Nitsche MA, Seeber A, Frommann K, et al. Modulating parameters of excitability during and after transcranial direct current stimulation of the human motor cortex. J Physiol 2005:568(1):291-303.

[11] Mulquiney PG, Hoy KE, Daskalakis ZJ, Fitzgerald PB. Improving working memory: exploring the effect of transcranial random noise stimulation and transcranial direct current stimulation on the dorsolateral prefrontal cortex. Clin Neurophysiol 2011;122(12):2384-9.

[12] Monte-Silva K, Kuo M-F, Liebetanz D, Paulus W, Nitsche MA. Shaping the optimal repetition interval for cathodal transcranial direct current stimulation (tDCS). J Neurophysiol 2010;103(4):1735-40.

[13] Abraham WC. Metaplasticity: tuning synapses and networks for plasticity. Nat Rev Neurosci 2008;9(5):387.

[14] Jasper HH. The ten twenty electrode system of the international federation. Electroencephalogr Clin Neurophysiol 1958;10:371-5.

[15] Bienenstock EL, Cooper LN, Munro PW. Theory for the development of neuron selectivity: orientation specificity and binocular interaction in visual cortex. J Neurosci 1982;2(1):32-48.

[16] Huang Y-Y, Colino A, Selig DK, Malenka RC. The influence of prior synaptic activity on the induction of long-term potentiation. Science 1992;255(5045):730-3.

[17] Nitsche M, Paulus W. Excitability changes induced in the human motor cortex by weak transcranial direct current stimulation. J Physiol 2000;527(3):633-9.

[18] Fricke K, Seeber AA, Thirugnanasambandam N, Paulus W, Nitsche MA Rothwell JC. Time course of the induction of homeostatic plasticity generated by repeated transcranial direct current stimulation of the human motor cortex. J Neurophysiol 2011;105(3):1141-9.

[19] Murakami T, Müller-Dahlhaus F, Lu M-K, Ziemann U. Homeostatic metaplasticity of corticospinal excitatory and intracortical inhibitory neural circuits in human motor cortex. J Physiol 2012;590(22):5765-81.

[20] Turrigiano GG, Nelson SB. Homeostatic plasticity in the developing nervous system. Nat Rev Neurosci 2004;5(2):97-107.

[21] Siebner HR, Lang N, Rizzo V, et al. Preconditioning of low-frequency repetitive transcranial magnetic stimulation with transcranial direct current stimulation: evidence for homeostatic plasticity in the human motor cortex. J Neurosci 2004;24(13):3379-85.

[22] Creutzfeldt OD, Fromm GH, Kapp H. Influence of transcortical D-C currents on cortical neuronal activity. Exp Neurol 1962;5:436-52.

[23] Fregni F, Boggio PS, Valle AC, et al. Homeostatic effects of plasma valproate levels on corticospinal excitability changes induced by $1 \mathrm{~Hz}$ rTMS in patients with juvenile myoclonic epilepsy. Clin Neurophysiol 2006;117(6):1217-27.

[24] Lang N, Siebner HR, Ernst D, et al. Preconditioning with transcranial direct current stimulation sensitizes the motor cortex to rapid-rate transcranial magnetic stimulation and controls the direction of after-effects. Biol Psychiatry 2004;56(9):634-9.

[25] Monte-Silva K, Kuo M-F, Hessenthaler S, et al. Induction of late LTP-like plasticity in the human motor cortex by repeated non-invasive brain stimulation. Brain Stimul 2013;6(3):424-32.

[26] Simis M, Adeyemo BO, Medeiros LF, Miraval F, Gagliardi RJ, Fregni F. Motor cortex-induced plasticity by noninvasive brain stimulation: a comparison between transcranial direct current stimulation and transcranial magnetic stimulation. Neuroreport 2013;24(17):973-5. 